DOI: https://doi.org/10.46296/yc.v5i9edespsoct.0109

\title{
EXPERIENCIA EDUCATIVA DE APLICACIÓN DEL DISEÑO UNIVERSAL DE APRENDIZAJE EN EL ECUADOR
}

\section{EDUCATIONAL EXPERIENCE WITH THE APPLICATION OF THE UNIVERSAL DESIGN FOR LEARNING IN ECUADOR}

\author{
Coppiano-Loor Carlos Cecilio ${ }^{1}$; Corral-Joza Karen Elisa ${ }^{2}$ \\ ${ }^{1}$ Universidad San Gregorio de Portoviejo USGP. Portoviejo Ecuador. Correo: \\ e.cccoppiano@sangregorio.edu.ec. ORCID ID: https://orcid.org/0000-0001-6394-6117 \\ ${ }^{2}$ Universidad San Gregorio de Portoviejo USGP. Portoviejo Ecuador. Correo: \\ kcorral@sangregorio.edu.ec. ORCID ID: https://orcid.org/0000-0002-8209-4084
}

\begin{abstract}
Resumen
El presente artículo proporciona una experiencia educativa a través de una investigación con enfoque cuantitativo. Se analizaron los conocimientos de los docentes sobre el Diseño Universal de Aprendizaje (DUA), sus aportaciones a la inclusión educativa y su implementación en el sistema educativo, considerando que el DUA se encuentra en una etapa de descubrimiento y reconocimiento en el Ecuador. De acuerdo con los principios metodológicos interpretativos y del problema a investigar, se situó en la investigación no experimental del tipo exploratorio descriptivo, a través de una recogida sistemática de los datos que admite el análisis descriptivo. Las técnicas utilizadas fueron documental y de campo, se realizó encuesta de opción múltiple dirigida a docentes, identificando el nivel de conocimiento del DUA en los procesos enseñanza aprendizaje; y una entrevista a una experta sobre las buenas prácticas del DUA en el país. Como resultado de la indagación sobre aportaciones exitosas del DUA estas son muy escasas en el sistema educativo ecuatoriano. Se propone, para que el Diseño Universal de Aprendizaje cumpla con sus principios y pautas, una formación masiva e integral a los docentes en esta metodología innovadora e incluyente.
\end{abstract}

Palabras claves: Diseño universal de aprendizaje; Diversidad; Necesidades educativas.

\begin{abstract}
This article provides an educational experience through a quantitative research approach. The knowledge of teachers about the Universal Design for Learning (UDL), its contributions to educational inclusion and its implementation in the educational system were analyzed, taking into account that the UDL is in a stage of discovery and recognition in Ecuador. According to the interpretative methodological principles and the problem to be investigated, it was placed in the non-experimental research of the descriptive exploratory type, through a systematic collection of data that admits the descriptive analysis. The techniques used were documentary and field, a multiple-choice survey was conducted among teachers, identifying the level of knowledge of the $S A D$ in the teaching-learning processes; and an interview with an expert on the good practices of the SAD in the country. As a result of the research on successful SAD contributions, these are very scarce in the Ecuadorian educational system. In order for the Universal Design for Learning to comply with its principles and guidelines, a massive and comprehensive training of teachers in this innovative and inclusive methodology is proposed.
\end{abstract}

Keywords: Universal Learning Design; Diversity; Educational Needs.

Información del manuscrito:

Fecha de recepción: 22 de julio de 2021.

Fecha de aceptación: 24 de septiembre de 2021.

Fecha de publicación: 01 de octubre de 2021. 


\section{Introducción}

A principios de los años 90, CAST (Center for Applied Special Technology) comenzó a investigar, desarrollar y articular los principios y pautas del DUA Pastor et al., (2013). Esta investigación centra en que la escasa aplicación del Diseño Universal de Aprendizaje afecta las habilidades de razonamiento de los estudiantes de octavo grado en la Unidad Educativa Juan Cruz Aizprúa, de la parroquia Boyacá, Manabí, Ecuador. La declaración de Salamanca, tiene como principio rector que las escuelas deben acoger a todos los niños, independientemente de sus condiciones físicas, intelectuales, sociales, emocionales, lingüísticas u otras, con el objetivo de dar una educación de calidad a todos con un enfoque integrado dentro de la sociedad que nos rodea. Duk, Cisternas, \& Ramos, (2019).

La Ley Orgánica de Educación Intercultural (LOEI), dentro de los principios generales, articulo 2 y sus literales, define que la educación es un derecho humano de aprendizaje permanente a lo largo de toda su vida con libertad y autonomía para su emancipación una educación en valores, en democracia, respeto a la diversidad étnica, social y la eliminación de toda forma de discriminación (Asamblea Nacional, 2011). La educación es dinámica y se encuentra en proceso de mejora continua, según lo que menciona la Ley Orgánica Reformada (LOEI) en su artículo 2.3.- Principios del Sistema Nacional de Educación, literal q. Diseño Universal de Aprendizaje: Ofrece flexibilidad al currículo en lo referente a las maneras en que todos los estudiantes accedan al aprendizaje, según sus fortalezas y necesidades (Asamblea Nacional, 2021).

Este artículo tuvo como objetivo indagar los conocimientos de los docentes sobre el Diseño Universal de Aprendizaje (en adelante, DUA) en el sistema educativo, por lo que resulta sustancial conocer cuál es la comprensión del DUA y qué estrategia didáctica aplica el docente identificada con tales principios en la labor educativa. Los docentes en sus prácticas pedagógicas aplican diferentes técnicas, estrategias didácticas, evaluaciones, pero que tanto estas estrategias didácticas son aplicadas para la diversidad de 
los estudiantes y cubrir las necesidades de aprendizaje de cada discente.

La Unidad Educativa Juan Cruz Aizprúa cuenta con una población estudiantil aproximada de 500 estudiantes que proviene de comunidades aledañas, con escuelas unidocentes y bidocentes donde el educador sin una correcta planificación de enseñanza no cumple con los objetivos básicos imprescindibles expuesto en el currículo educativo. En ese entorno, el DUA ayuda a los docentes a alcanzar este objetivo, proporcionando un marco para entender cómo crear estrategias didácticas que atiendan las necesidades de todos los estudiantes desde el primer momento, (Pastor et al., 2013).

EI DUA es un marco basado en la intervención del currículo con dimensión en la planificación de objetivos, métodos, materiales 0 recursos y evaluaciones, lo que permite múltiples formas de representación de la información, múltiples formas de expresión y acción del aprendizaje, múltiples formas de participación, ya que no existen dos personas iguales, no existe dos estudiantes que aprendan de la misma manera, esto hace que el DUA recoja esas diferencias y diversidad y la plasme en un marco practico para un aprendizaje significativo (Vázquez, 2020).

La educación inclusiva se concreta en las aulas atendiendo a la diversidad del alumnado, lo que supone el paso necesario para garantizar la igualdad de oportunidades en el acceso al proceso educativo. El atender las diferentes habilidades de adquirir la información de manera individual va a ser un proceso complejo, por lo que concluimos con la necesidad de capacitación docente en DUA como alternativa didáctica en el marco de la educación inclusiva al proporcionar las mismas oportunidades a todo el estudiantado independientemente de sus características individuales (Espada, Gallego, \& Gonzalez, 2019).

Definición del Diseño Universal de Aprendizaje y sus fundamentos neurocientíficos.

EI CAST tras estudios, llegó a la conclusión de que las tecnologías debían de ponerse al servicio de los estudiantes y no al revés y, que el 
problema o la dificultad no residía en los alumnos sino en el currículo, que no se adaptan a las diferencias individuales (Pastor et al., 2013).

Los fundamentos del DUA residen en las teorías del aprendizaje, en las tecnologías de la información y comunicación, y en la neurociencia, también conocido por muchos como neuroeducación. El DUA hace referencia de cómo funciona el cerebro y su aplicación en el aprendizaje. Los estudios con diagnóstico por imagen, han conseguido identificar 3 grupos de redes neuronales que intervienen en el aprendizaje, estas son (Vázquez, 2020):

1. Las que intervienen en la representación de la información: redes de reconocimiento.

2. Las que intervienen en la acción y la expresión de los aprendizajes: redes estratégicas.

3. Las que intervienen en la motivación y en la implicación para el aprendizaje: redes afectivas.

Su funcionamiento es modular, es decir, no intervienen de manera aislada e independiente, aunque si son específicas y/o especializadas. Este dato resulta relevante, se entiende que, si todas estas redes están conectadas y activadas, la calidad del aprendizaje que se produce es mejor, por ello este es uno de los retos que persigue el enfoque del DUA, optimizar el proceso y la calidad del aprendizaje (Pastor, 2016).

Existen tres principios fundamentales basados en la investigación neurocientífica que guían el DUA y proporcionan el marco para las pautas:

Principio I: Proporcionar múltiples formas de representación (el qué del aprendizaje)

Los estudiantes difieren en las diferentes formas percibir y adquirir el conocimiento dentro del proceso enseñanza aprendizaje distintamente de sus capacidades educativas especiales, unos adquieren la información de una forma más rápida a través de medios visuales, otros a través de medios auditivos, otros por medio de la practicidad, entonces no existe un mecanismo estático que me indique la única forma de adquirir la información. 
Principio II: Proporcionar múltiples formas de acción y expresión (el cómo del aprendizaje)

Los discentes son muy diversos a la hora de expresar sus análisis o criterios sobre un evento o situación dentro del aula, esto debido a muchos factores del medio por ejemplo: desarrollo social, medio escolar, estudiantes con alteraciones físicas, o problemas de lenguaje, etc. Unos pueden expresarse bien de forma oral, otros escrita, a través del arte, todas expresiones y acciones requieren de una gran cantidad de estrategias por parte del docente para reconocer las diferentes formas de expresarse de los estudiantes. En realidad, no hay un medio de acción y expresión óptimo para todos los estudiantes; por lo que proveer opciones para la acción y la expresión es esencial.

Principio III: Proporcionar múltiples formas de implicación (el porqué del aprendizaje)
El elemento primordial en el desarrollo escolar para el aprendizaje, "es la motivación" en todo el proceso educativo debe estar presente la implicación para aprender. Existen múltiples fuentes que influyen a la hora de explicar la variabilidad individual afectiva, como pueden ser los factores neurológicos y culturales, el interés personal, la subjetividad y el conocimiento previo, junto con otra variedad de factores presentados en estas pautas (Alba, 2018).

\section{Enfoque del Diseño Universal de aprendizaje basado en el currículo para desarrollar conocimiento.}

El DUA, además del enfoque que tiene sobre el currículo, busca que los estudiantes sean analíticos, críticos, reflexivos, que estén motivados en aprender más. En cuanto al currículo, los componentes en que se basa el DUA son cuatros que están interconectados y estos son (Vázquez, 2020): 
Tabla1. Vázquez, (2020).

\begin{tabular}{|c|c|c|c|}
\hline Objetivos & Métodos & Materiales & Evaluaciones \\
\hline $\begin{array}{l}\text { Son definidos } \\
\text { teniendo en cuenta } \\
\text { la variabilidad entre } \\
\text { los estudiantes y, } \\
\text { diferenciándolos de } \\
\text { los medios para } \\
\text { lograrlos. Esto } \\
\text { permite establecer } \\
\text { expectativas más } \\
\text { altas sobre las } \\
\text { posibilidades de } \\
\text { cada alumno. }\end{array}$ & $\begin{array}{l}\text { Se facilita una } \\
\text { mayor } \\
\text { diferenciación de } \\
\text { métodos en base } \\
\text { a la variabilidad } \\
\text { del alumno } \\
\text { respecto al } \\
\text { contexto de la } \\
\text { tarea, } \\
\text { caracterizan por } \\
\text { ser flexibles y } \\
\text { variados. }\end{array}$ & $\begin{array}{l}\text { Se brindan las } \\
\text { herramientas, } \\
\text { recursos y apoyos } \\
\text { necesarios para } \\
\text { permitir al alumno } \\
\text { acceder, } \\
\text { examinar, } \\
\text { organizar, } \\
\text { abreviar } \\
\text { exponer y } \\
\text { comprensión de } \\
\text { los conocimientos } \\
\text { de distintas } \\
\text { maneras. }\end{array}$ & $\begin{array}{l}\text { Se permite } \\
\text { adaptarse a la } \\
\text { variabilidad, con el } \\
\text { fin de reducir y } \\
\text { eliminar barreras } \\
\text { en la valoración } \\
\text { justa de los } \\
\text { conocimientos, } \\
\text { habilidades e } \\
\text { implicación de los } \\
\text { estudiantes. }\end{array}$ \\
\hline
\end{tabular}

Para que exista un verdadero enfoque aplicado del DUA se necesita poner atención y compromiso a estos cuatro elementos para que todo proyecto perdure y sea aplicable en la educación.

\section{Aportaciones exitosas del Diseño} Universal de Aprendizaje

Según (Abellan \& Sanchez 2013), las estrategias organizativas $y$ metodológicas deben contemplarse en las programaciones docentes y unidades didácticas para facilitar la adecuación del currículo a las características y necesidades de los discentes. Partiendo de esta premisa, se presenta una experiencia innovadora postulada en los principios del DUA, que tiene por objetivo el diseño, implementación y evaluación de las unidades didácticas en la que se incluyen diferentes medidas ordinarias de atención a la diversidad. Los resultados muestran el enriquecimiento del proceso de enseñanza-aprendizaje al insertar estas medidas en los materiales curriculares, así como la acogida positiva de la propuesta por parte de los discentes.

Durante las últimas décadas, la ansiedad constante de los docentes y profesionales en educación se ha centrado en cómo atender de manera equitativa a sus discentes (Gaviria \& Palacios 2020),, lo que exige que en el trabajo pedagógico que se desarrolle al interior del aula de clases, se ofrezcan diversas 
oportunidades para el conocimiento; de modo que permita integrar a todos los estudiantes, respetando sus ritmos y estilos de aprendizaje, que se evidencie durante todo el proceso de enseñanza aprendizaje el principio de equidad igualdad y diversidad. El verdadero desafío de las instituciones educativas $y$ profesionales de la educación es desarrollar estrategias didácticas creativas que implementen el DUA con el fin de proporcionar oportunidades de aprendizaje inclusivas, diversas para todos los estudiantes desarrollando al máximo sus competencias.

El DUA se coloca como una alternativa didáctica en el marco de la educación inclusiva. Expresa la necesidad de romper con el enfoque homogéneo de la educación habitual, que considera que todos los estudiantes son iguales y aprenden de la misma forma y que el rol docente es el de organizar y preparar los materiales con el mismo formato para todos, fomentado la exclusión antes que la inclusión educativa (Espada, Gallego, \& Gonzalez, 2019).

\section{Metodología}

La investigación tuvo un enfoque cuantitativo, desde este enfoque metodológico se consiguió analizar los conocimientos de los docentes sobre el DUA, detectar sus aportaciones a la inclusión educativa y su implementación en el sistema educativo.

De acuerdo a los principios metodológicos interpretativos, el problema a investigar se sitúo en la investigación no experimental, del tipo exploratorio descriptivo, estando el DUA en una etapa de descubrimiento y reconocimiento siendo flexible en la metodología aplicada, a través de una recogida sistemática de los datos que admite el análisis descriptivo.

Las técnicas utilizadas fueron documentales y de campo por medio de entrevista estructurada realizada a profesionales expertos que han investigado sobre el DUA y su incidencia en el aprendizaje de los estudiantes. También se realizó encuesta de opción múltiple dirigida a docentes de la Unidad Educativa Juan Cruz Aizprúa, identificando el nivel de conocimiento del DUA en los procesos enseñanza aprendizaje. 
La población objeto de estudio consistió a profesionales de la educación (docentes) de la Unidad Educativa Juan Cruz Aizprúa, un total de 30 profesionales los que fueron informados con el propósito del estudio, y con expertos que sustentaron la investigación a través de una entrevista sobre el DUA en el sistema ecuatoriano.

\section{Resultados}

De acuerdo con los resultados obtenidos el $50 \%$ de los docentes pretenden aplicar frecuentemente el DUA en las prácticas educativas. (Tabla 1). Según Abellan \& Sanchez
(2013), las estrategias organizativas y metodológicas deben contemplarse en las programaciones docentes y unidades didácticas para facilitar la adecuación del currículo a las características y necesidades del estudiantado. La mayoría de población encuestados manifiestan conocer sobre los enfoques del DUA mas no lo aplican desde los principios metodológicos establecidos por los autores, situación que impide un aprendizaje acorde a los ritmos y necesidades individuales de los estudiantes, respetando la diversidad al momento de aprender, tal como se manifiesta dentro del enfoque del DUA.

Tabla 2. Los docentes aplican el DUA en las prácticas educativas.

\begin{tabular}{clc}
\hline$\#$ & Alternativas & Porcentaje \\
\hline $\mathbf{1}$ & Muy frecuentemente & $10 \%$ \\
2 & Frecuentemente & $40 \%$ \\
3 & Ocasionalmente & $40 \%$ \\
4 & Raramente & $10 \%$ \\
5 & Nunca & $0 \%$ \\
\hline
\end{tabular}

Por otro lado, se identifica que el $66.7 \%$ de los docentes encuestados no conocen de los principios y pautas que utiliza el DUA en las prácticas educativas (Tabla 2). Espada, Gallego, \& González, (2019) concluyeron que existe una necesidad de capacitación docente en DUA como alternativa didáctica, en el marco de la educación inclusiva, al proporcionar las mismas oportunidades a todo el estudiantado independientemente de sus características individuales, desafíos para la formación del profesorado de 
todos los niveles educativos. Se constata así la importancia de desarrollar en los docentes competencias para el abordaje de la diversidad en el aula.

Los principios del DUA están basados en las redes cerebrales involucradas en el aprendizaje de manera proactiva, los docentes encuestados creen conocer los principios del DUA pero no lo aplican por ser un proceso creativo complejo y un nuevo paradigma didáctico. Desde el enfoque de las bases teóricas los múltiples medios de representación, diferentes maneras de enseñar y transmitir conocimientos, se promueve el establecimiento de conexiones entre los diferentes elementos de la información, temática que el docente aplica de forma homogénea para todos los estudiantes sin tomar en cuenta la diversidad de recursos y actividades que permitan el acceso y el aprendizaje para todo el grupo estudiantil. (Valencia \& Hernández, 2017)

El DUA parte de la diversidad desde el comienzo de la planificación didáctica, facilita a los docentes un marco para enriquecer y flexibilizar el diseño del currículo, reducir las barreras educativas y proporcionar oportunidades de aprendizaje a todos los estudiantes desde la base de sus limitaciones $y$ potencialidades.

Tabla 3. Principios y pautas que utiliza el DUA en las prácticas educativas

\begin{tabular}{|c|c|c|}
\hline \# & Alternativas & Porcentaje \\
\hline 1 & $\begin{array}{l}\text { Proporcionar múltiples medios y formas de: } \\
\text { representación, acción y expresión, e }\end{array}$ & $33.3 \%$ \\
\hline 2 & $\begin{array}{l}\text { Razonamiento deductivo, inductivo, hipotético- } \\
\text { deductivo, transductivo }\end{array}$ & $16.7 \%$ \\
\hline 3 & Redes de reconocimiento, estratégicas, afectivas. & $16.7 \%$ \\
\hline 4 & Razonamiento lógico, deductivo, inductivo. & $33.3 \%$ \\
\hline
\end{tabular}

El DUA es una metodología prometedora que abarca la diversidad de aprendizaje de los estudiantes dentro del aula de clase con la aplicación de los principios y pautas de aprendizaje. De este modo, el segundo principio del DUA tiene una presencia más profunda en los estudiantes. Según Sánchez, (2016) en cada principio, las pautas 
siguen una organización jerárquica vertical, desde lo más simple en términos de procesamiento cognitivo y de facilidad de implementación, hasta lo más complejo (recursos dirigidos a mejorar la comprensión, las funciones ejecutivas 0 la implicación activa en el proceso de aprendizaje). Y es que el principio 2 tiene más relación con la manera en la que los estudiantes pueden expresar lo que saben, sienten, conocen, adquiere o percibe la información "función ejecutiva", mientras que los principios 1 y 3 se dirige hacia los docentes el "qué" y el "porqué" del aprendizaje, es muy importante la profesionalización de los educandos en metodologías incluyentes ya que estos principios se centran en como ellos representan la información y motivan a los estudiantes en los procesos cognitivos.

\section{Conclusiones}

Como resultado de la indagación en conocimientos de los docentes sobre el DUA en el Ecuador, son muy escasas en el sistema educativo ecuatoriano una de las bases para que el DUA cumpla con los principios y pautas es la formación de los docentes en esta metodología innovadora e incluyente respondiendo a la diversidad dentro del aula, Las pautas van dirigidos en como el educador desarrolla la representación el "qué" del aprendizaje y la implicación el "porqué" del aprendizaje, atendiendo a la diversidad que se tiene en el aula y a las necesidades de los discente y el "cómo" del aprendizaje en las funciones ejecutivas de los estudiantes.

El principio de flexibilidad y creatividad es lo que menciona en la entrevista desarrollada a la sub secretaria de educación especializada e inclusiva del Ministerio de Educación del Ecuador, "sobre el DUA y su aplicación en el sistema educativo", hace énfasis en las competencias que deben fomentar los docentes para la atención a la diversidad y los estilos de aprendizaje dentro del aula, clases más dinámicas que utilicen muchos recursos en la representación de la información, considerando los estilos de aprendizaje, ser un buen planificador, dinámico, flexible considerado los intereses y 
necesidades de los estudiantes, otras de las competencias es la implicación o motivación, el trabajo en equipo totalmente tanto en los docentes, DECE, UDAI y todos los actores educativos, gestionar y propiciar nuevas formas y fórmulas de evaluación que parte también del DUA, potenciar el trabajo autónomo de los estudiantes, desarrollar ambientes de trabajo cooperativo,

Los docentes creen conocer los principios del DUA mas no lo aplican por ser un proceso creativo complejo y un nuevo paradigma didáctico. Desde el enfoque de las bases teóricas los múltiples medios de representación, temática que el docente aplica de forma homogénea para todos los estudiantes sin tomar en cuenta la diversidad de recursos y actividades que permitan el acceso y el aprendizaje para todos los educandos, es aquí donde aparecen las pautas del DUA, que no son herramientas o estrategias nuevas, si no que ya son conocidas por la mayoría de los profesionales educativos. Por lo tanto, se debe indicar que uno de los objetivos del DUA es reducir las barreras 0 dificultades de acceso al currículo y atender las necesidades de todos los estudiantes desde el inicio de su etapa educativa.

\section{Bibliografía}

Abellán, A., \& Sánchez, A. (2013). UNA EXPERIENCIA DE INNOVACIÓN EN EDUCACIÓN PRIMARIA: MEDIDAS DE ATENCIÓN A LA DIVERSIDAD Y DISEÑO UNIVERSAL DEL APRENDIZAJE. Murcia: Universidad de Murcia.

Alba, et al. (2018). Pautas sobre el Diseño Universal para el Aprendizaje (DUA). Madrid: Universidad Complutense.

Constituyente, A. (2008) Constitución de la República del Ecuador. Quito: Ecuador.

Coral, A. L. (2012). DESARROLLO DE HABILIDADES DE PENSAMIENTO $Y$ CREATIVIDAD COMO POTENCIADORES DE APRENDIZAJE. San Juan de Pasto, Colombia: Universidad Mariana.

Cristina, V. P., \& Osvaldo, H. G. (2017). El Diseño Universal para el Aprendizaje, una alternativa para la inclusión educativa en Chile. Cuba: Universidad de Matanzas Camilo Cienfuegos.

Duk, C., Cisternas, T., \& Ramos, L. (2019). Formación Docente desde un Enfoque Inclusivo. A 
25 Años de la Declaración de Salamanca, Nuevos y Viejos Desafíos. Chile: Revista Latinoamericana de Educación Inclusiva.

Espada, Gallego, \& González. (2019). Diseño Universal del Aprendizaje e inclusión en la Educación Básica. Ecuador: Revista de educación Alteridad.

Espada, Gallego, \& GonzálezMontesino. (2019). Diseño Universal del Aprendizaje e inclusión en la Educación Básica. Revista de educación Alteridad, Universidad Politécnica Salesiana, Ecuador., 10,11.

Gaviria, I. C., \& Palacios, I. M. (2020). Diseño universal de aprendizaje y currículo. Chile: Universidad Arturo Prat de Chile.

Nacional, A. (2011). Ley Orgánica de educación Intercultural. Quito: Asamblea.

Nacional, A. (2021). Ley Orgánica Reformatoria de la Ley Orgánica de Educación Intercultural. Quito: Asamblea Nacional.

Pastor et al. (2013). Pautas sobre el Diseño Universal para el Aprendizaje (DUA). Madrid: Universidad Complutense de Madrid.
Pastor, C. A. (19 de 9 de 2016). Berritzegune Nagusia. Obtenido de https://www.youtube.com/wat $\mathrm{ch} ? \mathrm{v}=\mathrm{x} X \mathrm{Xxe} 1 \mathrm{nc} 7 \mathrm{kQ}$

Sánchez, et. al. (2016). Análisis Factorial de las Percepciones Docentes sobre Diseño Universal de Aprendizaje. Chile: Universidad de Burgos.

Vázquez, M. D. (2020). Valoración de diversos profesionales educativos sobre el DUA y la inclusión educativa. España: UNIVERSITAT OBERTA DE CATALUNYA. 